\title{
THE NANOWORKBENCH: Automated Nanorobotic system inside of Scanning Electron or Focused Ion Beam Microscopes
}

\author{
Ivo Burkart, Volker Klocke and Eva Maynicke
}

Klocke Nanotechnik GmbH, Pascalstr. 17, 52076 Aachen, Germany

Many every day developments would not exist today without preparation, handling and assembly of materials under optical microscopes. There would be no wristwatch, no in vitro fertilization, no minigearbox, just to mention a few. These products depend on using toolsets like tweezers, knives, hooks, probes and several different measurement tools in combination with optical microscopes, since a good part of the understanding about material functions and process technologies was developed this way. But material properties and functionalities also depend on structure dimensions that are smaller than the wavelength of light.

The operators of SEM, FIB or Dual Beam systems generally work without toolsets, although the wavelength limit of light is no physical boundary for using such tools. One reason for this is the disconnected closed loop operation between human eyes and hands that enable complex operations under optical microscopes without even thinking about it.

The Nanoworkbench is the first system substituting this eye-hand coordination effectively with nanoprecision in a SEM/FIB-system. It can be imagined how technology could evolve, when tools within a SEM/FIB can be used as easily as tools used under optical microscopes. The two main aspects of this new system, the development of its Nanorobotics technology and the applications enabled by it, are described in this paper.

Aspect 1, development of the technology: In general the success of in-SEM/FIB Nanorobotics depends on the co-operation of several important modules in one global system. The main developments include:

- Nanomanipulators in automation, for movement of end-effectors, sample handling and preparation,

- $\quad$ Several different end-effectors for nano- probing, cutting, cleaning, force distance or wear measurements, gripping, sorting or material preparation and processing,

- $\quad$ Automatic in-situ tip cleaning process, e.g. for continuous nano-manipulation or nano-cutting,

- $\quad$ Automatic 3D position detection of all tools and SEM/FIB-samples up to 3D sample topography measurements,

- A control of all tool and SEM/FIB sample stage positions in a common global coordinate system, - SEM picture assisted haptic interface by "Live Image Positioning",

- Modular design for fast configuration \& teaching of nano-analytical or nano-handling processes,

- $\quad$ One common automation control for Nanorobotics and SEM/FIB.

With instantiating these technical demands the Nanoworkbench enables the secure and easy usage of toolsets within SEM/FIB systems, for manual operation, for non-professional users and in high level of automation, e.g. for high throughput industrial processes, even as job-shop [1].

Aspect 2, development of a series of new applications in one system: Expanding the SEM/FIB to a material processing system and a nano-analytical workbench opens the door to new applications, from material research over biology \& bionics, pharmacy, tribology, environmental geology, forensic research and semiconductor technology up to nanofabrication and production [6]. Several examples of these new interdisciplinary research and development fields will be described during the presentation, together with the invitation to participate at an actual research network forming further new applications 
by proof of concept studies.

A few examples of Nanoworkbench applications are highlighted in FIG 1. Although these examples may raise the impression of a review about different machines and their usage, this is not the case. Described is the development of the Nanoworkbench.

\section{References:}

[1] D. Morrant, EIEx Magazine of European Innovation Exchange, 1 (2009), p. 6.

[2] G. Schmid, M. Noyong, Colloid Polym Sci., (2008) DOI 10.1007/s00396-008-1866-2

[3] Seong Chu Lim, Keun Soo Kim, Kay Hyeok An, Department of Physics, Sungkyunkwan University, Korea (2002)

[4] Described in detail at www.3D-Nanofinger.com

[5] C.-H. Ke1, H.D. Espinosa, Journal of the Mechanics and Physics of solids, 53 (2005) p. 1314

[6] Supported by European Commission, IST and Ziel2.NRW, see: www.nanohand.eu, www.hydromel-project.eu, www.nanowerkbank.com

Figure 1. includes the rows:

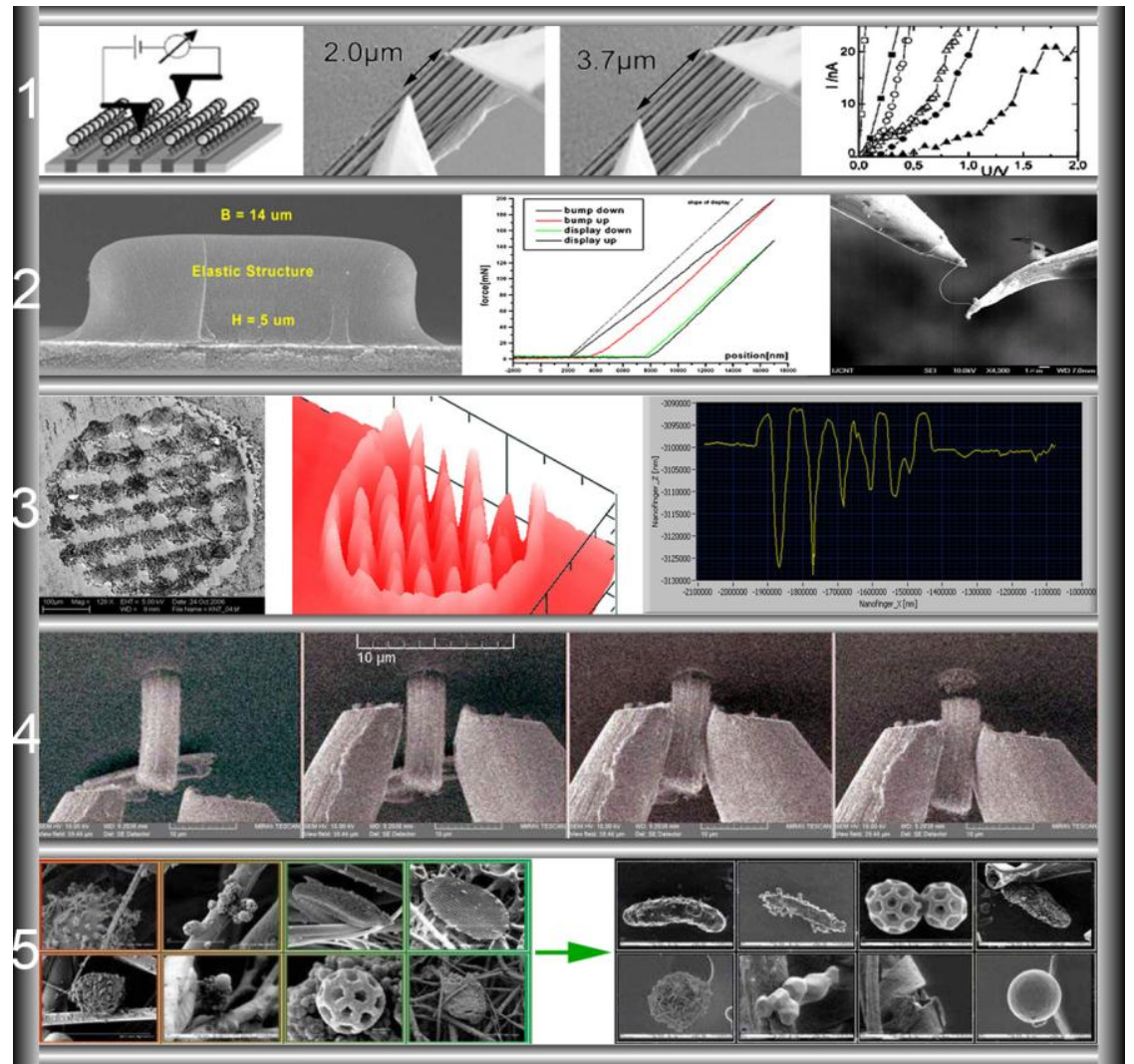

1) Nano-Probing of Gold55-Clusters arranged in chains [2]: The electrical conductivity along these gold chains is measured over different distances and compared with the conductivity of bare gold wires.

2) Nano-tribology and similar measurements can be performed in high resolution [3].

3) SEM image and 3D-Topography measured quantitatively by the "Dimensional SEM" module [4].

4) Gripping of a rigid CNT bundle with high force and separation from the ground [5],

5) Particle sorting from source area (left image set) to clean target area (right image set) 